\title{
LA QUESTIONE DEL MISTERO UMANO NELLA FILOSOFIA DELLA FORMAZIONE
}

\section{The question of the human mystery inside the philosophy of formation}

\section{La question du mystère bumain dans la philosophie de la formation}

\section{Paola PAscucci}

Università degli Studi di Genova. Dipartimento di Studi Umanistici. Corso Andrea Podestà 2-16128. Genova (Italia). Correo-e: paspao72@libero.it

Fecha de recepción: enero de 2009

Fecha de aceptación definitiva: abril de 2009

Biblid [(1130-3743) 21, 1, 2009, 49-70]

\section{SOMMARIO}

In questo lavoro, la oopía (sophía) e la Bildung sono interpretate come i due cardini di un discorso che riguarda l'essere umano inteso come possibilità significante. Bildung e Umbildung vengono problematizzate in relazione al significare del mistero umano. La questione del mysterium hominis è trattata, all'interno della filosofia della formazione, in riferimento al significato originario del greco mystèrion sino all'interpretazione pedagogica dell'esperienza ebraico-cristiana del Mistero di Dio (Mysterium Dei).

Parole-chiave: filosofia, Bildung, Umbildung, mýo-sfera, mistero, mistificazione, verità, dubbio, ironia, stupore, teologia mistica. 


\section{SUMMARY}

In this work, the oopía (sophia) and the Bildung are interpreted as the two poles of a speech which regards the human being understood as a significant possibility. Bildung and Umbildung gets problematic in relation to the meaning of the human mystery. The question of mysterium bominis is dealing, inside the philosophy of formation, referring to the original greek meaning of mysterrion until the christianhebrew experience of uncreated Mystery (Mysterium Dei).

Key words: philosophy, Bildung, Umbildung, mýo-sphere, mystery, mystification, truth, doubt, irony, astonishment, mystical theology.

SOMMAIRE

Dans cette oeuvre, la oopía (sophía) et la Bildung sont interprétées comme les deux pivots d'une discussion qui concerne l'être humain entendu comme possibilité signifiante. Bildung et Umbildung sont rendues problématiques par rapport au signifié du mystère humain. La question du mysterium hominis est traitée, dans la philosophie de la formation, par rapport à la signification originelle du grec mystèrion jusqu'à l'expérience judéo-chrétienne du Mysterium Dei.

Mots clés: philosophie, Bildung, Umbildung, mýo-sphère, mystère, mystification, vérité, doute, ironie, stupeur, théologie mystique.

1. Mistero e filosofia della formazione: architettura della Questione

La pedagogia si configura come "la scienza generale della formazione e dell'educazione dell'uomo" (Gennari-Kaiser, 2000, 11). La questione del mistero riguarda il sapere pedagogico perché il mysterium sostanzia la pienezza della formazione umana.

La densità semantica della parola Bildung (Gennari, 1995b) riconduce al mysterium hominis da cui diparte una raggiera di interrogativi che, anche all'interno dell'indagine pedagogica, stilizzano l'architettura di una quaestio antica ma non antiquata, resistente come irìdio ed ancóra iridèscente. Rapportato al mistero creato -che gli pertiene, ma a cui non perviene- ed inscritto nel Lógos increato, l'uomo significa ciò di cui è Bilde. Qui il significare è connesso, aristotelicamente, al $\lambda$ ó $\gamma \mathrm{s} \varsigma$

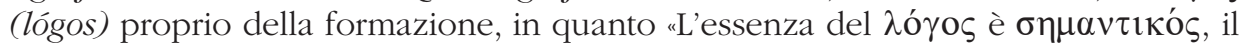
significare» (Heidegger, 1983, 405).

In altri termini, il mistero concerne l'integralità dell'essere umano in quanto significante di una dimensione intrinseca ed altra, la quale può dischiudersi al Mistero divino -ovvero al superamento della temporalità e della finitezza in un oltre connesso all'Origine-. 
Bildung e Umbildung in relazione al significare del mistero -proprio dell'essere umano -sembrano invocare identità ${ }^{1}$, evocare alterit $\grave{a}^{2}$, provocare trascendenza, che appunto "significa trascendenza nel mistero" (Rahner, 1967-69, 403). Questo termine, riconducibile al mystèrion greco e alla sua trascrizione nel latino mysterium -sino alla cristianizzazione nel Mysterium Christi-, si può delineare come il luogo dell'identico, dell'altro e dell'oltre. Dunque, in quanto luogo della formazione, il mistero dell'uomo interpella le scienze umane e si sottrae a determinazioni storiche e sociologiche, antropologiche ed etnografiche, psicologiche e psicoanalitiche, teologiche e filosofiche. Il velo del mistero creato non viene lacerato dalla penetrante acuità dell'indagine scientifica. Il decrescere del quoziente di comprensione del mistero in sé riconduce, per definizione, alla sua inespugnabilità.

Ciò nonostante, la questione inerente il mysterium hominis -in virtù del suo porsi supra non contra rationem- persiste in quanto scaturigine di problemi ultimi circa l'incipit, la forma e l'exitus dell'esistenza dell'uomo in formazione e trasformazione. Non di rado, il tema del mysterium risulta banalizzato, popolarizzato e assediato da una nebulosa preconcettuale, invece di essere frequentato dagli esiti di un serio impegno euristico -nonché dalla scèpsi affinché- in pedagogia -nessun perimetro epistemologico diventi parametro di verità dogmatica-.

La dimensione antropologica, in senso ontogenetico e filogenetico, presuppone la quaestio del mistero, che una imparziale problematizzazione della Bildung non può trascurare. Se lo facesse si otterrebbe la compromissione dell'integrità dell'indagine concernente la formazione, poiché quest'ultima fruisce di forme -naturali, culturali, tradizionali- ${ }^{3}$ le quali riconsegnano l'uomo al principio e fondamento di quel percorso esistenziale, che dall'identico si apre all'altro fino al collasso della misura nell'Incommensurabile.

Lo svolgimento del $\lambda$ ó $\gamma$ os (lógos) -inteso come "il dire sulle cose" (Heidegger, 1983, 51) - deve poter incontrare/scontrare la scientia della formazione dell'essere umano che liberamente si forma pensandosi e, altrettanto liberamente, si pensa formandosi (Gennari, 2001, 24-25). Pertanto, dove e in che modo l'uomo individua il Grund per pensarsi e formarsi? Come può illudersi di affrancare se stesso -essendo in grado di scegliere, perfino, una tensione deformativa- dal fondo della sua formazione e del suo pensiero? La oọ́i (sophía), a questo riguardo, è uno dei cardini del discorso libero sulla formazione, del lógos aperto -alla maniera di una

1. Pensare la pedagogia significa interrogarsi circa l'identità uomo-formazione. A questo riguardo Gennari (2001, 744) rileva: "Prima della pedagogia, in quanto scienza, c'è quella identità: l'uomo è la sua Bildung. Ma Bildung non è soltanto formazione. E' relazione, fondazione, incontro, trasformazione, libertà, natura, mondo, vita, sentimento, mistero. Immagine del mistero! Immagine della formazione: Bild della Bildung!”.

2. Il mistero dell'altro è, in fondo, il mistero dell'altro di sé, dell'altro da sé e dell'Altro in sé. Quest'ultimo è concepito da Origene come il Mistero di Dio che, nel Verbo, sposa la natura umana.

3. Per quanto concerne l'approfondimento delle categorie di natura, tradizione e cultura nell'àmbito della formazione umana si rinvia allo studio di KAISER (1998). 
soglia- di fronte all'"essere-in-possibilità" (Gennari-Kaiser, 2000, 50) dello homo bumanus. Riconoscendosi fondato, l'uomo conosce se stesso come possibilità

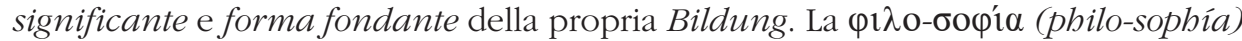
della Bildung sembra porre in rilievo la concavità del pensare la formazione e la convessità del formare il pensare, all'interno della volontà di rigore e stenìa epistemologica.

La filosofia della formazione, dunque, non sussiste come ancilla philosophiae allo stesso modo in cui la filosofia non è ancilla theologiae; il che equivale ad una dichiarazione di pariteticità in nome di quella oopía (sophía) concepita come primo cardine della soglia del pensare e del formare.

Il secondo cardine di quella soglia è rappresentato dalla Bildung. Se la formazione è "sempre l'uomo stesso" (Rosenzweig, 2000, 186), la Bildung dovrebbe riguardare un uomo capace di essere oltre per essere ontologicamente se stesso. In tal senso, la formazione potrebbe essere letta anche come corrispondenza intrinseca di ossimori grondanti mystèrion.

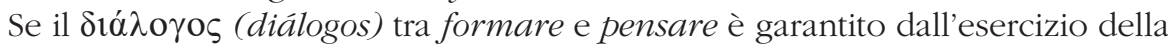

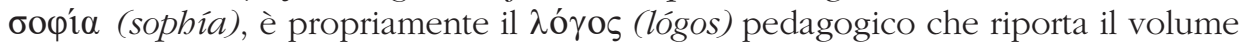
dialogico all'essere umano, il quale concretamente si forma e si pensa in quanto

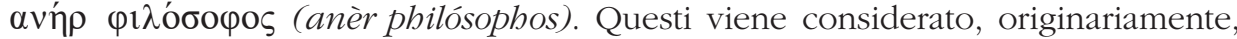

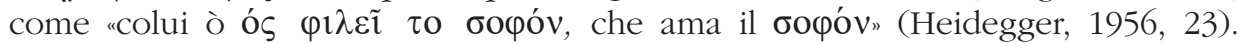
Tale uomo ama la sapienza nel senso eracliteo della corrispondenza sapiente al

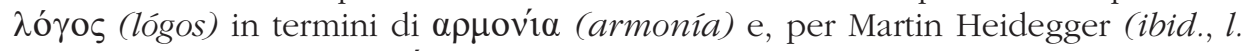
c.), davvero "questa $\alpha \rho \mu$ víl $\alpha$ è ciò che caratterizza in modo peculiare il $\varphi \imath \lambda \varepsilon \tilde{\imath} v$, e cioè l'amare". Individuato nel tempo e nello spazio l'uomo si ritrova, nondimeno, esposto alla malattia mortale di memoria kierkegaardiana in quanto dispera di poter essere se stesso, alla stessa maniera in cui dispera di non potersi liberare per divenire altro da sé. Inoltre, l'essere umano è esibito al rischio di contrarre ed incubare -all'opposto- una sorta di malattia immortale che lo inscrive nello spazio del mistero, il quale -lungi dall'essere sentito e vissuto- viene di fatto subito come enigma.

È a questo punto che la nudità disperante della finitezza lo incalza e lo muove, ancor di più, a negarsi come possibilità significante.

L'essere umano rimane così serrato nell'insolubilità dell'enigma pur desiderando, disperatamente, di poter essere oltre da sé. In fondo, cos'è la disperazione se non una alterazione della speranza? Bisogna ribadire che l'uomo disperato non ha età perché "essenzialmente la disperazione di un tal giovane e di un tal anziano è la stessa: non si arriva a una metamorfosi nella quale prorompe la consapevolezza dell'eterno, dell'io, perché possa cominciare la lotta la quale o eleva la disperazione a una forma più alta o conduce alla fede" (Kierkegaard, 1991, 68). La fede devitalizza questa alterazione. L'uomo di speranza crede nonostante tutto, superando-si.

Allo stesso modo in cui "Soffrire è vivere senza poter vivere" (Valéry, 1942, 52), così l'estraniazione del mistero dalla formazione -che interpella anche lo studioso dal fondo della sua umanità- conduce l'uomo al vivere senza poter 
soffrire quella acuminata inquietudine che conduce alla ricerca ontologica di sé. Proprio la mozione euristica previene dallo scacco dell'essere formato rispetto alla dignità di un formar-si che possa dirsi inquieto -e non disperato-, libero -e non disimpegnato-, rigoroso -e non rigido-, assertivo -e non asservente-. Il mistero umano rimanda, d'altronde, all'imprevedibilità, al doute ${ }^{4}$ (Rimbaud, 1975 , 28-29), al pulsare aritmico dell'ignoto nel noto. Perciò esso è rintracciabile -ma non riducibile- ad alterazioni ematochimiche ${ }^{5}$. Non è caratterizzabile raccogliendo unicamente i frammenti coscienti filtrati dalla fessurazione dell'inconscio. Non si esplica nel riferimento alle costellazioni dell'irrazionale e dell'esoterico. Non risulta, d'altra parte, esauribile nella divulgazione convinta del nulla di Dio all'interno di un uomo vinto dal nulla.

Alla pienezza del mistero -distinto dalle sue approssimazioni: quali enigma, occulto e magia- pare associarsi la vuotezza del nulla che diverge sia dall'apofatismo medioevale -dall'affondare in gotis nicht (Eckhart, 1999)-, sia dalla visione della vacuità funzionale alla rientificazione (Ichtung) delineata dallo studioso giapponese Keiji Nishitani.

Mistero e nulla-come i pieni e i vuoti di una stessa architettura-concorrono nel sagomare una questione che parla dell'uomo, il quale esiste e diviene attraverso la progressiva assunzione, privazione e mutamento di forme nel tempo e nello spazio. Obiettivo di questo lavoro è, dunque, individuare -attraverso la sophía della Bildung- il dinamismo di un mistero operante, indicando le cifre razionalizzanti e segnalando i depotenziamenti prescientifici intorno all'umano essere-inpossibilità, che vibra nel luogo spirituale della formazione. Infatti, nella magna silva di codificazioni improprie del mysterium si cercherà un sentiero etimologico e l'orizzonte di una chiarità a matrice filosofica e teologico-mistica.

Come l'architettura di un edificio riconduce a chi vi risiederà, così l'architettura pedagogica della questione del mistero riconduce a uno tra i possibili dubbi che sembrano far affiorare l'uomo -che è la sua formazione- da se stesso: sarà l'essere umano a dover tutelare il proprio mistero o sarà il Mistero -che trascende $i$ misteria preservare l'umano dal moderno processo di nientificazione?

4. L'uomo intende leggere, nell'opalescenza del mistero, il senso dell'umana finitezza, ma come scrive Arthur Rimbaud: "Le Doute nous punit! / Le doute, morne oiseau, nous frappe de son aile... / - Et l'horizon s'enfuit d'une fuite éternelle!...”. Per la traduzione italiana Rimbaud (1975, 29).

5. Ci si riferisce alla variazione della concentrazione idrogenionica in relazione al fenomeno dell'alcalosi, intesa come abbassata concentrazione di ioni idrogeno con $p H>7,45$. In particolare, agli effetti temporanei che sembrano indotti dall'alcalosi da iperventilazione polmonare e alla sua ricaduta sullo stato di coscienza. Montinari (1998, 116-117). 


\section{IL PROBLEMA DEL MYSTEERION: COORDINATE E PRECISAZIONI TERMINOLOGICHE}

In primis, si rende necessaria l'attivazione di un doppio processo - di chiarificazione e demistificazione- riguardante una estesa mýo-sfera, concepibile come l'area comune riguardante quei vocaboli (quali mistero, misterioso, misterico, mistagogico, misteriosofico, misticista e mistico) che si raccordano, probabilmente, al greco $\mu$ $\omega$ (mýo). La dimensione del chiudere per custodire qualcosa -a cui riconduce semanticamente il verbo $\mu$ v́ (mý) - è evocata, ad esempio, da espressioni linguistiche come $\mu$ vińpiov (mystèrion): che rimanda al significato

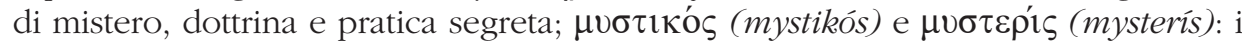
quali richiamano rispettivamente l'arcano e la mistica; $\mu$ v́o $\tau \varepsilon \varsigma$ (mýstes): che rende

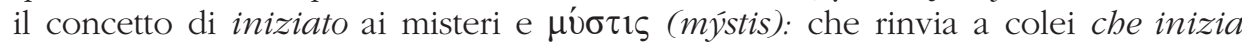
ai misteri; $\mu \nu \sigma \tau \imath \tau o \lambda \varepsilon v ́ \omega$ (mystipoléuo): il quale riporta all'azione del celebrare i misteri. Nell'elaborare il termine "mistero" in quanto voce dizionariale nell'ambito tematico della teologia biblica, Jean-Noël Aletti ${ }^{6}$ specifica che la parola greca mystèrion "comprende un radicale (la radice muô) e una desinenza (-tèrion). La finale (-tèrion) sembra indicare un senso originale locale, ossia strumentale" (Aletti, 2005, 856). Questa precisazione concernente il radicale $\mu$ v́ $\omega$ (mýo) e la desinenza - $\tau$ ṕp v permette di far notare la correlazione tra mys-tèrion e religioni misteriche, a partire dalle quali il mistero, per Aletti (ibid., l. c.), "avrebbe inizialmente designato il luogo d'iniziazione, e poi i riti come anche i segreti, cioè i misteri [...] celebrati". In particolare Luigi Borriello ${ }^{7}$, nella Presentazione all'opera di Bouyer (1998: 5), non rileva solo che la parola mysterion deriva da myo. Nell'ambito veterotestamentario, difatti, questo termine che "rende l'ebraico sod (= conversazione confidenziale), traduce l'aramaico raz acquistando un preciso significato teologico: si tratta di un mistero escatologico, cioè della visione anticipata di ciò che nel progetto di Dio dovrà accadere" (ibid., l. c.).

Il plurale greco mystèria -sottolinea Nicolas Derrey ${ }^{8}$ alla voce mistero nel contesto della teologia liturgica e sacramentaria studiata con precisione nell'ambito delle Chiese di lingua aramea e siriana- traduce il termine râzê di matrice persiana e dal significato enigmatico. Infatti, "Il senso di enigma di tali râzê permane, ma s'impone l'idea dell'attesa manifestazione, specie quando il plurale râzê designa i riti destinati a mostrare e a donare ciò che essi nascondono. Ne risulta un'accentuazione escatologica della liturgia" (Derrey, 2005, 857). Viene dunque còlta una correlazione tra linguaggio pagano e riti cristiani in concomitanza all'opzione linguistica e alla ineguale fruizione semantica del singolare greco

6. All'interno di Lacoste (1998) Per la traduzione italiana, a cui riferire le citazioni, Aletti (2005, 856-857).

7. Nella Presentazione della traduzione italiana dell'opera di BouYer (1986). Nel presente articolo, i riferimenti citazionali sono tratti dall'edizione italiana datata 1998.

8. DeRrey (2005, 857-859). 
mystèrion -tradotto con il latino mysterium e riconducibile a ciò che resta segretoe del plurale mystèria, rimasto immutato perché riferito alla specifica dimensione cultuale misterica di matrice pagana.

Nel contesto dello studio delle Chiese di lingua latina e riferendosi a Mohrmann, Derrey afferma -riguardo all'adozione del termine misteri nel cristianesimo- che "la traslitterazione del plurale mystèria, che pure era disponibile, venne accuratamente evitata durante i primi tre secoli» (Derrey, 2005, 858). Si è cercato, quindi, di non ammettere nella sfera linguistica latina le parole greche giudicate dubbie a causa della tacita densità sacrale originaria. In altri termini, vengono messi da parte "gli equivalenti latini dei termini greci designanti le azioni sacre e l'esperienza cultuale delle religioni basate sui misteri (sacra, arcana, initia, ecc.), per adottare sacramentum e sacramenta" (ibid., 858-859). Tale ponderatezza può essere correlata alla fascinazione esercitata dai misteri e alla massima fedeltà degli iniziati, che ne custodivano la segreta rivelazione nel pieno silenzio. Infatti, «il segreto delle religioni misteriche non è che il segreto dei loro riti” (Bouyer, 1998, 35): Anzi, si mostra essenziale interrogarsi sulla veridicità dell'idea stessa di religioni misteriche. Infatti, sembra legittimo "chiedersi se si siano mai avuti altri "misteri", nel senso originale del termine, nell'ellenismo se non quelli di Eleusi" (ibid., 36).

Una sfera linguistica in movimento diacronico e dall'ampio raggio è, dunque, implicata nella determinazione della questione circa il mistero dell'uomo a cui si associa sia la problematizzazione della realtà religiosa -e delle azioni di culto ad essa connesse-, sia la concettualizzazione del sacro in generale. In un capitolo dello studio di Price ${ }^{9}$ viene tracciato il ruolo del cittadino greco (esattamente in età infantile e prepuberale come pure in relazione alla differenza di genere) nell'ámbito rituale.

Simon Price (2002, 117-125) segnala alcune cerimonie. Viene indicata la celebrazione, nel mese Pianepsione, della festa pubblica delle Apaturie

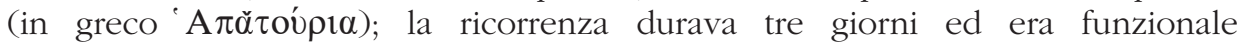
all'immissione dei giovani nelle fratrie. Sono poi individuate nel mese estivo Sciroforione, la suggestiva festa delle $\alpha \rho \rho \eta$ nobili prepuberi le quali, prescelte dall'arconte, portavano in processione gli arredi sacri ad Atena Polias, nonché il rito ateniese delle vergini consacrate ad Artemide Brauronia detto delle arktoi, che è il plurale del femminile "orsa" -traducibile con il termine á $\rho \kappa \tau о \varsigma$ (árktos)-. Il significato di quest'ultimo rituale, solennizzato con cadenza quadriennale, è reso intelleggibile solo adottando una lente mitologica e religioso-cultuale. Viene, inoltre, ricordato che il matrimonio, spazio di educazione della prole, non era consacrato -come si potrebbe pensare fruendo di categorie religiose in termini etnocentrici, ma caratterizzato con modalità differenti sempre su base mitico-rituale.

9. PrICE (1999). Qui ci si riferisce alla traduzione italiana PRICE (2002, 117-136) . 
Risulta interessante nella esposizione di Price (2002, 132-136), la successiva problematizzazione del rituale e, soprattutto, la descrizione del luogo sacro

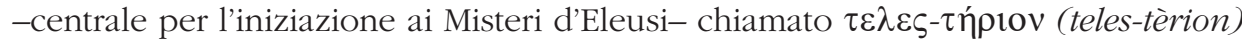
il quale, come si è già indicato riferendoci a Jean-Noël Aletti, riconsegna il termine mys-tèrion alla desinenza - $\tau$ ńpiov (-tèrion). A questo riguardo, si aggiunge che la radice $\tau \varepsilon \lambda \varepsilon \zeta$ (teles) riconduce al verbo $\tau \varepsilon \lambda \dot{\varepsilon} \omega$ (teléo), il quale pare associare semanticamente l'inizio ai misteri all'idea di compimento perfetto. Si profila, a questo punto, l'esigenza di accennare la relazione (o l'eventuale divaricazione) tra la dimensione iniziatica di matrice pagano-ellenistica e la moderna ricerca della verità.

\subsection{La forma del mistero: indicibilità, invisibilità, velatezza}

La tutela del segreto impone l'attribuzione di un velo. Proprio il binomio velamento-svelamento sembra essere quella forma specifica sostanziante il nucleo dell'iniziazione ai misteri -e di quelli "per eccellenza" (Bouyer, 1998, 37) rappresentati dai misteri eleusini- che vanno preservati dallo sguardo indagatore e superficiale dei profani.

Il significare delle cerimonie iniziatiche riporta, dunque, a ciò che deve essere velato per non risultare disperso nelle ridondanze dell'arte del parlare, a cui Plutarco -eclettico discepolo del platonico Ammonio e poi sacerdote del santuario

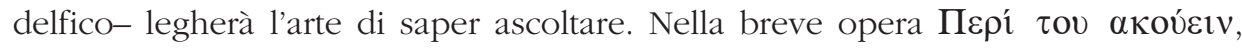
composta poco prima del 100 d.C., l'autore dei Moralia espone quest'arte al figlio di Eutidamo, il quale aveva da poco cessato di fare uso della toga puerile. Plutarco spiega, allora, al giovane Nicandro che è «solo seguendo la ragione che si può essere veramente liberi" e, riferendosi al filosofo Spintaro, puntualizza che non risultò mai essere di facile individuazione qualcuno il cui sapere fosse più del suo parlare (Plutarco, 2006, 55). Pertanto, seguendo Plutarco, si può aggiungere che l'ascolto è possibile solo a chi, esercitandosi nel tacere, ha assimilato profondamente il controllo della parola: la regola del silenzio. Soprattutto di fronte al mistero, il silenzio possiede una funzione di raccoglimento. Al rischio della dispersione di senso si oppone la concentrazione semantica in quell'area del $\mu$ ó (mýo) in cui il tacere significa la nudità delle parole di fronte a ciò che è segreto ed in cui il linguaggio è soltanto il raffinato abito dell'indicibile, comunque significante. D'altra parte, alla correlazione tra gli opposti dicibilità/indicibilità si associa il nesso oppositivo visibilità/invisibilità.

Infatti, il silenzio misterico, riconducibile agli iniziati, è legato alla consistenza di ciò che deve essere velato per non essere profanato. Il velato va custodito come tale in funzione di quella conoscenza che riguarda una élite. Non a caso, si parla di conoscenza perché l'amante della sophía -cioè il filosofo- è generalmente iniziato ai misteri, come sottolinea Agathias nell'approfondimento de "La mistica greca". 
Nelle pagine dedicate a "I misteri e il sistema iniziatico", Leucon Agathias ${ }^{10}$ nota che "nelle cerimonie dei misteri, gli iniziati ordinari, per quanto ci è dato di sapere, non vedevano che statue velate o vestite, mentre gli alti dignitari avevano il diritto di vedere le statue nude; l'abbigliamento è qui paragonato ai veli che ricoprono la verità" (Agathias, 2000, 85). La verità si mostra agli iniziati divenuti degni di vedere la nudità. In tal senso, la velatezza diventa risorsa di quella ricerca veritativa che si esprime nell'azione combinata -tra $\varphi 1 \lambda \varepsilon \tilde{i} v$ (phileín) e $\sigma o \varphi^{\prime} \alpha$ (sophía) - annessa al filosofare sull'uomo, il quale è la sua formazione. Inoltre, nel Novecento, proprio in relazione alla verità come "svelatezza", alla velatezza come "non svelatezza" e, dunque, alla velatezza come "non-verità", Martin Heidegger parla di mistero. Nelle lezioni del 1929-1930 -dedicate ai Die Grundbegriffe der Metaphysik- Heidegger (1983, 41) considera la verità come "preda che deve venir strappata alla velatezza", proprio in riferimento al lógos disvelante, il quale trae fuori dalla velatezza "il prevalere dell'ente nella sua totalità" e dice la $\alpha \lambda \eta ́ \theta \varepsilon ı$ (alétheia), la quale si configura come quanto è non-velato. Il filosofo di Friburgo, come è noto, costruisce progressivamente la questione della fine e del superamento della filosofia prima.

La parola metafisica viene definita non originaria -in quanto non "si è formata a partire da una esperienza umana essenziale e originaria come sua espressione"

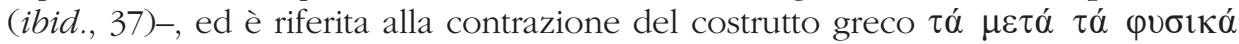
(tá metá tá physiká). Heidegger argomenta sul "prevalere dell'ente nella sua totalità" e, perciò, sul termine púøis (phýsis) che «in quanto questo ente nella sua totalità, non è inteso nel senso moderno e tardo di natura, magari come concetto opposto a quello di storia, bensì in un modo più originario, in un significato che viene prima di natura e storia e abbraccia entrambi questi concetti e in un certo modo include

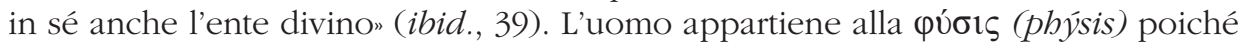
l'esistenza dell'uomo esprime tale prevalere, d'altra parte, anche il lógos le appartiene perché se "esprimere si dice $\lambda \dot{\varepsilon} \gamma \varepsilon \imath \mathrm{v}$ : il prevalere portato ad espressione è il $\lambda$ ó $\gamma \mathbf{o} \varsigma_{\text {" }}$ (ibid., l. c.). Precisamente, il lógos disvela pur tendendo a velarsi-come Heidegger rileva citando l'aristocratico filosofo di Efeso: Eraclito- perché il lógos appartiene al physikós. Inoltre, Heidegger annota l'álpha privativo che accompagna il termine $a$-létheia in attinenza al corrispondente $u n$ in lingua tedesca. Emanuele Severino ${ }^{11}$ specifica, in riferimento alla verità, che il termine tedesco "Un-verborgenheit (nonvelamento) è appunto il termine che esprime la struttura speculativa del termine a-létheia. Unverborgenheit esprime l'originaria unità dell'Entbergung (l'atto di svelare) e dell'Entborgenheit (il carattere dell'esser svelato dell'ente)". Profilando la comprensione greca della verità come preda, Heidegger $(1983,42)$ puntualizza che il prefisso privativo álpha -in relazione al significato riferito dal radicale nei

10. Agathias (1977). Si cita l'edizione italiana del primo volume dell'opera di Davy (2000).

11. Si rimanda ad alcune significative pagine del contributo monografico di Severino, riportate in Appendice nell'ámbito dell'edizione italiana del testo di Heidegger $(1999,81)$. 
composti della lingua- "esprime il fatto che alla parola dinnanzi alla quale è posto, manca qualcosa. Nella verità l'ente viene strappato alla velatezza" (ibid., l. c.).

L'estrema acuità speculativa con cui coglie che la verità -in quanto preda e quindi in quanto predabile e predata- "richiede l'impegno totale dell'uomo" (ibid., 43), non consente, però, al filosofo Heidegger di lasciarsi interrogare dalla presenza dell'uomo nella propria verità di uomo umano (Gennari, 2001, 669-684).

Nell'ampio dipanarsi di una scépsi antimetafisica e antiteologica, inoltre, la riflessione heideggeriana sembra rimanere imprigionata nelle fitte maglie che riconducono al nodo non districato della differenza ontologica. Heidegger non si sottrae alla questione del mistero, il quale viene correlato alla problematizzazione della verità e della libertà.

Ma a questo punto ci si potrebbe chiedere: la libertà può essere autenticamente pensata (e formata!), in senso heideggeriano, come Wesen della verità? Nel ragionare circa l'essenza pura della Wahrheit, Heidegger riprende il concetto tradizionale

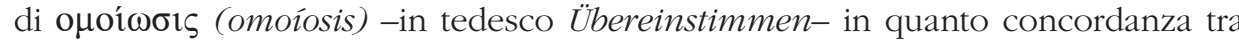
"asserzione" e "cosa" e ne specifica la condizione -che chiama "fondamento"- proprio nella libertà. Se l'essenza della verità è la libertà, l'essenza della libertà viene di fatto condotta al "lasciar-essere l'ente" (Heidegger, 1999, 43). Il filosofo tedesco spiega che questo lasciare essere non deve essere interpretato come espressione di una passività indifferente, ma va riferito -al contrario- all'essere coinvolti "da ciò che è aperto", da ciò che è svelato, in breve da quella $\alpha$ - $\lambda \eta ́ \theta \varepsilon 1 \alpha$ (a-létheia) la quale si configura come ciò che è non-velato.

La verità (Wahrheit) si contrappone alla non-verità (Un-wabrheit) che non può non essere che velamento. Puntualmente, configura la velatezza come "più antica anche dello stesso lasciar-essere che, svelando, già tiene velato e che sta in relazione con il velamento" (ibid., 47-48); proprio qui viene situato il quid che permea l'esser-ci. Ciò che permea l'esser-ci è, pertanto, il mistero inteso come "il velamento di ciò che è velato" (ibid., 48).

Ebbene, proprio il mistero distoglie l'umanità errante perché Heidegger concepisce la non-verità come "erranza" fuorviante e, contemporaneamente, non fuorviante. In ultima analisi, se l'uomo storico è governato dalla costituzionale erranza dell'esser-ci, nella speculazione heideggeriana, il mystèrion dell'uomo umano non può che essere predato alla maniera della verità -ma anche depredatoperché dominato soltanto dal "mistero dell'esser-ci" (ibid., 50). Tuttavia, il mysterium bominis continua ad offrirsi libero allo sguardo euristico, poiché -in fondo- la verità "non è un disvelamento che distrugge il mistero, bensì una rivelazione che gli rende giustizia" (Benjamin, 1972-1989, 73).

Se -come insegnano i maestri medioevali- l'oggetto di una quaestio può essere tutto quello che si riconosce come problema da disputare, resta legittimo chiedersi che cosa significa "fare problema" in riferimento al mystèrion che, per definizione, rimane tale.

Si ribadisce che il focus della questione del mistero nella filosofia della formazione non può essere rappresentato dal tema del mystèrion in sé -il quale 
risulta impermeabile, eppure significante in senso filosofico, esperibile in senso teologico-mistico, pletorico in senso poetico- quanto sulla realtà umana associata al mistero. Difatti, con varie declinazioni semantico-linguistiche -concisamente ricondotte alla polarità dicibilità/indicibilità e al nesso oppositivo visibilitàl invisibilità, ovvero riportate al tacere degli iniziati greci e allo svelamento proprio della verità di cui tratta Heidegger- l'essere umano ha sempre tentato di veicolare il significare del suo mistero, senza mai dissolverne la consistenza vitale e liquefarne la solidità dialettica.

\subsection{Il mistero del problema: prossimità e approssimazioni al Mystèrium}

La parola Gelassenheit viene pronunziata nella filosofia heideggeriana e, da questa, sottratta alla mistica speculativa medioevale eckhartiana.

Per indicare il luogo dell'abbandono (Zur Erörterung der Gelassenbeit), si assiste al colloquio significativo tra uno Scienziato, un Erudito e un Maestro. All'interno di questo scambio paritetico, il Maestro comprende che "Per il bambino che è racchiuso nell'uomo la notte resta sempre Colei che approssima le stelle» (Heidegger, 1959, 77). Ma è inaspettatamente l'Erudito a spronarci verso un pensiero che produce pensare: "La meraviglia potrebbe allora dischiuderci ciò che è chiuso?". Inoltre, "ciò che è chiuso" appartiene automaticamente ad una oscura nebulosa? In altri termini, ciò che è serrato proviene dalla sfera del mýo nei termini di contrapposizione alla ragione? Se così fosse perché questa sfera è soggetta al dubbio e alla necessità di discernere e di precisare i significanti che la popolano? In ultima analisi, il mistero non potrebbe essere formato dall'uomo stesso in quanto essere culturale e spirituale? E ancora, l'uomo si può realmente trasformare in relazione a un mistero che, culturalmente, si presenta come il Mistero increato? Forse, il mistero inerisce "un re ignoto il cui regno era lo strano Regno degli Interstizi,? (Pessoa, 2002, 31).

La lettura dell'Oltre-Dio di Fernando Pessoa -che si definisce multiplo in virtù dell'ampia fruizione di eteronomi- non può che pungolare una certa curiositas. Anzi, l'Além-Deus di questo poeta del Novecento può incalzare il tentativo di ripensare criticamente la dimensione occulta ed esoterica, non sovrapponibile al Mistero e alla meraviglia dell'uomo di fronte ad esso. Nel ventre rigonfio della produzione letteraria pessoana gorgoglia un segreto fluire di correnti a matrice gnostica, ermetica, rosacrociana e massonica. Eppure, non si può negare che quella superficie esoterica permette di far luccicare sull'uomo che si forma una sorta di bagliore meglio precisato da Pessoa -nella A queda-come Clarão de Desconbecido.

Di fatto, il contenuto della lirica Deus risuona ancora in questi termini: "A volte sono il Dio che porto in me [...] non sono altro se non un ateo di questo dio che sono quando mi esalto. Vedo in me tutto un cielo ed è solo un alto cielo vuoto" (Pessoa, 2002, 35). In quelle poesie intitolate rispettivamente No tumulo de Christian Rosencreutz e Natal, Pessoa approda poi all'inquietante affermazione per 
cui "Dio è l'Uomo di un altro Dio maggiore" (ibid., 105) ed esorta a: "Non cercare e non credere: tutto è occulto" (ibid., 53). L'enigmaticità di tale invito -esito della convinzione pessoana per cui tutto è occulto- non riesce però a celare il fatto che l'occulto non è tutto, che l'assurdo è sempre in agguato e che l'ironia, a questo riguardo, può divenire un raffinato gioco di fioretto a funzione demistificante. Ma che rapporto esiste tra il dubbio, l'ironia e la formazione umana?

Il dibattuto significato del dèmone socratico - di "quel segno divino" (Platone, 1995, 38) che viene sentito dal maestro di Platone- sembra associabile alla ricerca della verità e allo smascheramento della non-verità. Nondimeno, il binomio ricercasmascheramento, la quale sospinge la dialettica ironico-maieutica, espone Socrate all'accusa infamante di avviare nei giovani un processo deformativo, di corrompere «i giovani germogli» (ibid., 37) rispetto alle credenze tradizionali della $\pi \mathrm{o} \lambda \iota \varsigma$ (pólis) antica. Socrate manifesta il coraggio di pensare autonomamente che cosa è il santo e, indirettamente, destabilizza la teologia mitologica antropomorfica la quale deve essere trasmessa in età giovanile tramite un'educazione adeguata. Come è noto, il dialogo socratico intitolato Eutifrone viene ambientato in prossimità del portico ateniese dell'Arconte re dove si incrociano un filosofo e un indovino, appunto Socrate ed Eutifrone. L'incontro del tutto casuale -ambientato nella sede in cui si celebrano delle azioni giudiziarie pubbliche connesse alla dimensione religiosasi rivela l'occasione per aprire un dialogo sulla questione del santo, producendo un netto confronto tra l'arte di ragionare del filosofo e la tecnica preconcettualemitologica dell'indovino.

Accusato pubblicamente di empietà dal giovane carrierista Meleto, Socrate perdura come il Maestro che provoca l'interlocutore. Quest'ultimo si trova nello stesso luogo di Socrate in quanto accusatore del padre -il quale in diritto di punire un suo servo omicida ne aveva causato accidentalmente la morte-. Eutifrone persiste nell'adesione alle credenze tradizionali e, alla fine, non può che sfuggire alla insistenza pressante del lógos di Socrate, il quale vuole -insieme a lui- "riesaminar da capo che cosa sia il santo" (ibid., 86).

Il movimento centrifugo verso un "altrove" -cioè il dover andare in un altro luogo, addotto come excusatio nei confronti del filosofo- abbandona Eutifrone sul piano del proteiforme. L'indovino, alla maniera del dio Proteo, si schermisce cambiando sembianza, prendendo forme diverse, perché non intende mettersi in gioco di fronte ad un pensiero pensante e formante. La maieutica socratica si approssima in ogni caso ad Eutifrone -che è più abile di Dedalo (ibid., 70-71) nel far muovere i discorsi- perché questi possa sostare nella propria ignoranza non per risiedervi, ma per riconoscerla. Anzi, nell'Epilogo, il Maestro greco vorrebbe quasi coartare il multiforme Eutifrone a non darsi per vinto, ad interpellarsi nuovamente sul problema centrale del dialogo, affinché questi non assuma il preconcetto del santo (saturo della traditio teologico-mitologica greca!) in quanto concetto dal carattere assiomatico. L'ironia socratica sembra connessa all'umiltà del Maestro che sa provocare e, contemporaneamente, lasciar-essere l'altro pur nell'amarezza procurata da una mancata speranza di formazione: "Che fai, o 
amico? Tu mi togli la grande speranza che nutrivo: di imparare da te che cosa è santo e che cosa no. [...] E avrei potuto anche vivere meglio per il resto della mia vita" (ibid., 86-87).

Dunque, non si dimostra vera l'affermazione pessoana per cui tutto è occulto perché l'occulto non è tutto. All'opposto, l'esercizio del dubbio -che va distinto dall'amaro abuso della diffidenza- e dell'ironia socratica -che mai si distilla nel vetriolo del sarcasmo- non devono abbandonare la formazione umana insieme a quello "stupore" che viene configurato da Martin Heidegger -in particolare nel testo

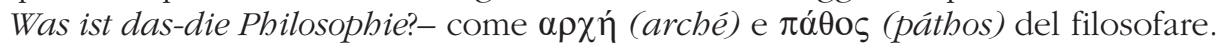

Difatti, è proprio lo stupore che origina e muove al pensare e al formare; in tal senso, uno stupore originario dell'uomo di fronte al suo mistero invia a quello medesimo stupore che percorre l'infanzia del filosofare (Aristotele, 1999, 46-47). Lo stupore fondamentale si scopre nel "mistero dell'infanzia" (Gennari, 2001, 325) allo stesso modo in cui "Nell'uomo vive l'infanzia del mistero-ovvero la sua parte più avvicinabile, sebbene coerentemente inconoscibile" (ibid., 324-325). Lo stupore attraversa l'infanzia della filosofia, si ravvisa nel mistero dell'infanzia e pulsa nell'infanzia del mistero umano. In altri termini, lo stupore parla di un mistero di armonia che si comunica nel silenzio mediante una flatus vocis (Bologna, 1992, 74-75) che gli è propria in senso archetipico e spirituale. La segreta lingua "des choses muettes" cantata da Baudelaire riguarda il poeta, ma anche il bambino il quale -dal mondo "delle cose mute"- approda sulle sponde del mondo parlato. Così, "Essere "ammessi" nel mondo è, parrebbe, "emettere" un suono ritmico, di cui, appunto, tace addirittura la parola del Poeta. Il $\mu \tilde{v} \theta \mathbf{o} \varsigma$ sostituisce il $\lambda$ ó $\gamma \mathbf{o}$ " (Bologna, 1992, 77).

Non a caso, il termine $\mu \tilde{v} \theta$ os (mýthos) è ricondotto da Bologna, a quella sfera del $m y ́ o$, a cui si lega la parola mistero. Quando Hölderlin, trattando della filosofia dello spirito, afferma che "Il filosofo deve [...] possedere un'attitudine estetica pari a quella del poeta" (Hölderlin, 1996, 162), sembra sorprendere nella bellezza la scaturigine dello stupore fondamentale.

Se "il supremo atto della ragione, quello con cui essa comprende la totalità delle idee, è un atto estetico, e che verità e bontà sono intimamente fuse soltanto nella bellezza" (ibid., l. c.), se platonicamente la bellezza è lo splendore del vero, d'altronde bisogna precisare, con Evdokìmov, che non tutto quello che è bello è anche vero. "In ogni bellezza c'è un segreto lutto" ricorda Walter Benjamin (1972-1989, 351). Infatti, la bellezza si profila -prima di essere circonfusa da un'aurea religiosa- come una sfera metafisica che si concede ad una misteriosa ambiguità e "che offre la più grande libertà. La sua potenza ammaliatrice affranca da ogni costrizione; all'éros della creazione si oppone l'éros della distruzione» (Evdokìmov, 1972, 61). Nell'estetica hölderliniana, "Monoteismo della ragione e del cuore, politeismo dell'immaginazione e dell'arte" (Hölderlin, 1996, l. c.), diventano l'apparato di quella religione che viene concepita in quanto "'estrema, la più alta opera dell'uomo" (ibid., 163). Ma l'uomo -che capitola in un credo "sensibile» (ibid., 162) ed autoreferenziale- non si asservisce forse ad una "fede idolatra nel potere 
teurgico e magico dell'arte» (Evdokìmov, 1972, 59), la quale contraddice l'idea "prima" hölderliniana consistente nella rappresentazione di sé "in quanto assoluta, libera essenza" (Hölderlin, 1996, 161)? Come è possibile essere liberi -o continuare ad esserlo- adorando un idolo fatto ad immagine dell'uomo tormentato che lo foggia?

Dostoevskij sembra ispirare la nitida teologia della bellezza evdokìmoviana nel riferimento platonico alla salvezza del mondo per opera della bellezza stessa. Eppure la bellezza della sua arte non sembra riscattarlo dalla cupa ombra di se stesso. Un pregevole studio sulla questione del Doppio viene elaborato da Otto Rank tramite un registro interpretativo analitico ed estetico. Der Doppelgänger viene richiamato, non a caso, da Freud nel lavoro intitolato Das Unheimliche. Rank studia le codificazioni dello scrittore russo -con Hoffmann, Heine, Maupassant, Lenau e Poe- in funzione dell'individuazione di una struttura psichica comune agli artisti. Rank -citando Merežkovskij che "considera il problema del Doppio come essenziale nell'opera di Dostoevskij" (Rank, 1914, 64)- ribadisce, proprio nella costruzione dei personaggi dostoevskiani, la tragicità delle parti di un tutto, le quali si inseguono per conseguire l'integrità perduta.

Inoltre, nell'affrontare il tema dell'immortalità dell'anima in attinenza all'ombra della morte, che sostanzia la letteratura e il folklore, questo allievo di Freud -tra l'altro segretario della società psicoanalitica viennese e direttore dell'Internationale Zeitschrift für Psychoanalyse- sostiene che "La credenza primitiva nell'anima, dunque, non è altro che una specie di credenza nell'immortalità volta a smentire energicamente la potenza della morte [...] L'idea della morte diventa sopportabile se c'è un Doppio che dopo questa vita ce ne assicura una seconda" (ibid., 102).

In tal senso, per Otto Rank, DerDoppelgänger possiede una funzione anticipatrice ed esorcizzante. Il doppio anticipa la morte e la esorcizza nell'anima individuata come duplicato del corpo. Nondimeno, l'affilata analisi rankiana -insensibile allo stupore improvviso dell'uomo vivente di fronte al mistero trascendente- prescinde dalla catarsi verticale dell'arte. Ad esempio, all'interno della prima parte dell'opera I fratelli Karamàzov (Brat'ja Karamazovy), precisamente nelle interpretazioni mistiche dell'inferno e del fuoco infernale (Dostoevskij, 1964, 408-409), se è vero che l'inferno viene definito come "Una sofferenza di non poter più amare", d'altro canto si riconosce all'essere umano "la facoltà di dirsi: io sono e io amo", ovvero il dono di un momento di amore vivente. Nel racconto del 1877 -Il sogno di un uomo ridicolo- è custodito il tragico monologo di un uomo che intende arrendersi alla propria volontà di morte per liberarsi dall'indifferenza e dall'umano abbandono.

Dal fondo di uno stato onirico -razziato dall'acuità dell'angoscia esistenzialeil protagonista è però capace di sentire la vita e, alla fine di questo fantastičeskij rasskaz, di dichiarare di non voler credere che il male possa costituire la condizione normotipica dell'umanità, perché l'immagine "vivente" della verità gli ha colmato l'anima (Dostoevkij, 1995, 84-85). Se è indubbio che la negazione dello Heimliche freudiano è la negazione di ciò che è noto all'uomo, allora il Doppelgänger si presenta come la pesante attestazione di quella negazione perché riporta alla fine 
dell'esistenza, non certo a quell'ultimo télos che significa fine, ma anche "confine" -come ricorda Karol Wojtyła nel saggio integrativo dedicato alla Trascendenza della persona nell'agire e autoteleologia dell'uomo-.

A differenza dell'occulto e della magia, le quali investono e manipolano una realtà esterna, il non-familiare agita le profondità dell'essere umano. Le radici di quanto è non-noto penetrano negli strati del sottosuolo psichico, eppure non riescono ad avvinghiarsi al mysterium bominis, pur rievocandolo. Piuttosto, a tratti, sembrano tradirlo perché -se la sfera estetica può ricondurre all'ambiguità- la sfera psichica tende al disconoscimento di quella integrità, che la bellezza prova comunque a ghermire. Solo la bellezza pare emettere quella duplice modulazione, sensibile e metafisica, che può aprire al religioso.

Difatti, quando l'uomo si scontra con i fantasmi intrapsichici non può che involgersi nella propria oscurità e, a volte, abbandonarsi all'angoscia dell'essere nel dolore per la morte, come viene mostrato nella analisi psicoanalitica rankiana della letteratura sul Doppelgänger.

Alla maggiore compenetrazione della vita corrisponde, per Friedrich Nietzsche, un uguale approfondimento del dolore. Nell'opera Also Sprach Zarathustra, il vedere l'abisso è legato al coraggio; tale visione concerne chi "guarda l'abisso, ma con fierezza" e lo "adunghia [...] con artigli d'aquila". In tal senso, nella vertigine del dolore e del male, l'uomo può ribadire il suo "essere-in-possibilità" (Gennari e Kaiser, 2000, 50).

Il mistero della morte riconduce l'uomo all'umanità che lo fonda. L'impatto con la finitezza sembra produrre una sorta di vuoto in cui trova spazio l'intensità, non solo emotiva, delle esperienze destabilizzanti. Così, il mysterium significa nel fondo dell'essere umano e indica una possibilità di attiguità ontologica (non solo di mero contatto psicologico!) in termini di identità, alterità e trascendenza. Sicché, dei "Chiari del bosco" dimorano nell'umano, con quello stupore classico che principia al filosofare.

La codificazione speculativo-poetica di María Zambrano, inoltra in quei claros che sono espressione di un "centro" - a volte inaccessibile e, comunque, osservabile "dal limite". Zambrano propone il doppio codice degli "scuri" e dei "chiari". I claros propiziano quella visibilità che permette la visione all'interno dell'oscurità, la quale raccoglie la luce e, pertanto, sottrae quest'ultima dalla dispersione.

L'intellettuale spagnola riporta la scissione della combinazione essere-vita alla perdita angosciante del centro Soltanto attraversando i chiari, si rimane sorpresi dall'improvviso "batter d'ali del senso" - dalla parola del bosque-, ovvero da quella "parola vera che proprio perché tale non può essere né interamente compresa né dimenticata" Se -nel mistero dell'amore creaturale- il soggetto è libero di dislocar-si come la "palabra sin ecos" ("parola senz'eco") cantata da Neruda quando si rivolge all'amata "silenciosa" - nelle maglie dell'enigmatico- non può che allontanar-si.

Occulto, esoterismo ed ignoto parlano di questo allontanamento e, pur evocandolo, non riescono a proferire il mysterium hominis. L'uomo che si pensa e si forma umanamente non disdegna, comunque, di avvicinare l'umana inquietudine, 
ossia quella nudità di se stessi un tempo confessata francamente dal poeta latino Gaio Valerio Catullo (1999, 145): "Odi et amo. quare id faciam, fortasse requiris. nescio, sed fieri sentio et excrucior" ("Odio e amo. Mi chiedi come si può. Lo sa il mio cuore crocefisso. Io non lo so").

\section{La Filosofia della formazione e il Mysterium nella cultura ebraico-Cristiana}

Nell'ámbito della filosofia della formazione se l'uomo è la sua Bildung e se la formazione è Gebeimnis (Gennari, 2001, 708-712), è necessario approfondire la gittata della relazione uomo-formazione-mistero. Se l'essere umano è la sua formazione, ogni uomo sta in essa come tale. In altri termini, l'uomo permane identico a se stesso nel Grund della propria umanità; in virtù di quest'ultima, l'essere umano si pone in relazione rispetto all'altro di sé e anche all'altro da sé. Pertanto, si riconosce come essere altro e con l'altro proprio rispetto al fondamento del suo essere uomo. La formazione, in quanto mistero, implica l'identità -perché l'uomo risiede nella sua umanità come uomo- e l'alterità-poiché è proprio grazie all'umanità di cui è depositario che l'essere umano si può approssimare all'altro $d i$ sé e da sé, in senso specie specifico, ovvero in modo pienamente umano-.

Ciò nonostante, se la Bildung si giocasse soltanto sul piano bicolore dell'identità e dell'alterità, allora si cederebbe allo scacco di una orizzontale linearità esistenziale, la quale espunge il mistero dell'uomo e della sua formazione e, quindi, converte la spinta verticale della Bildung nei termini esclusivi di formazione psicologica e sociale, comunque implicite.

L'essere umano è libero a tal punto da poter accettare o voler scegliere -anche a proprio svantaggio- il condizionamento e l'adattamento al posto della formazione, la quale è Umbildung (Sola, 2003). Rimane il fatto che l'uomo non è condizionato ontologicamente. L'uomo può superare se stesso in riferimento al suo essere spirituale (Kaiser, 1998), ossia al codice spirituale inscritto nella umanità che gli pertiene. La formazione incontra il mistero sino a delinearsi come mysterium dell'uomo. A questo punto, pare addensarsi un antico problema: sarebbe legittimo delineare l'uomo come mistero creato in relazione al Mistero increato, configurante l'essere umano come tale?

Nel Cherubinischer Wandersmann, Scheffler scrive: "L'aureità produce oro, la divinità Dio: Se non sei uno con essa, rimani piombo e fango" (Silesius, 1675, 150). In pieno Seicento, il sacerdote cattolico Johannes Scheffler -divenuto dapprima dottore philosophiae et medicinae all'Università di Padova e, in seguito, ricordato come Angelo della Slesia- elabora una interessante opera epigrammatica. Gli "Ingegnosi aforismi e rime che conducono alla contemplazione di Dio" sono pregni della timbrica mistica speculativa medioevale di indubbio riferimento eckhartiano, il quale non esclude l'influenza della mistica affettiva cinquecentesca di matrice spagnola, soprattutto, sanjuanista. Più che inscritti nel barocco tedesco 
della Slesia protestante, gli aforismi del Wandersmann si distinguono per l'afflato profondamente spirituale evocante un $\mu v \sigma \tau \imath \kappa o ́ s$ (mystikós), ormai cristianizzato.

Il riferimento alla mistica silesiana -la quale codifica la mistica eckhartiana in termini poetici- permette di cogliere il passaggio dal mistero dell'uomo al Mistero del Verbo, ovvero di problematizzare la presenza del mysterium hominis nel mysterium fidei.

Nel distico 250, contenuto nello Erstes Buch, Angelus Silesius celebra in versi quella divinità che produce Dio - die Gottheit machet Gott- allo stesso modo in cui l'aureità produce oro - die Goldheit machet Gold. Come si può inferire, nella visione mistica silesiana è l'unità con la Gottheit che libera Dio dalla sua determinazione ideale e sottrae l'essere finito alla finitezza. L'essere umano viene ricondotto al Grund che lo sostanzia in senso ontologico. Tale essere uno nell'Uno non riguarda i misteri oppure un mistero, ma concerne la mistica.

A questo riguardo, si impone una concisa precisazione del concetto di mistica, sia in senso generale che specifico, al fine di poterne cogliere il senso all'interno della problematizzazione del mistero e della formazione umana. La mistica non nasce come vocabolo autoreferenziale. Nel corso della storia, questa parola ha subito un processo di decontrazione dal refero teologico, addizionando -a partire soprattutto dal XVII secolo- significati eterogenei, spesso involti in una caligine semantica. E' da quel periodo che si assiste alla flessione concettuale e terminologica della mistica nella sua approssimazione misticista. Invero, l'aggettivazione mystica emerge in rapporto al termine teologia. Non si può pensare rigorosamente la mistica prescindendo dalla connessione dei due termini nella complementarietà originaria indicata nell'espressione teologia mistica. Da ciò si può inferire che -se l'area della mystica rimanda alla mýo-sfera - il termine theologia risulta attribuibile semanticamente all'antico discorso eracliteo, platonico e plotiniano, che riguarda il divino. Nell'elaborazione della voce "mistica" ", il significato di tale parola viene circoscritto all'ambito propriamente cristiano sottolineando che questa parola "ha perduto ogni precisione da quando J.-J. Rousseau ed i romantici l'hanno applicato a tutto l'irrazionale, spesso attribuito alla realtà religiosa" (Huot de Longchamp, 2005, 859).

Se si pensa la mistica come contemplazione all'interno di una configurazione estesa -che la restituisce al significato greco di $\theta \varepsilon \omega \rho i ́ \alpha$ (theoría) e del verbo $\theta \varepsilon \omega \rho \varepsilon ́ \omega$ (theoréo) - allora è possibile intuire che l'irrazionale non può riguardare la dimensione mistica allo stesso modo in cui l'antropomorfizzazione mitologica del divino le è estranea.

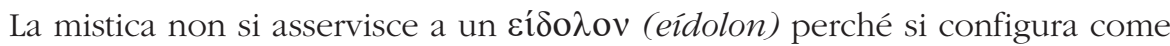
"aggettivo di "teologia", e il sostantivo ha ben marcato in sé il conio di lógos, almeno nella sua origine greca, platonica e aristotelica, dove nasce proprio in

12. Huot de Longchamp $(2005,859-865)$. 
opposizione ai miti e alle fantasie dei poeti sugli dèi” (Vannini, 1999, 9). Inoltre, la mistica richiama una nudità radicale nel rapporto di unione dell'uomo con il divino che, per grazia, lo rende eckhartianamente, dunque cristianamente, figlio nel Figlio. L'essenzialità portata all'estremo è la cifra della mistica, intesa esattamente come "Vita dello spirito" (Vannini, 2000, 7).

La consistenza teologica della nozione di Spirito permette al vero mistico di resistere a configurazioni preconcettuali come a rigide determinazioni concettuali -le quali, rivestendo il "nulla divino" (Eckhart, 1999), spogliano del carattere di unione il legame religioso con Dio-. A ciò si associa la possibile scissione tra l'elemento mistico e religioso in quanto il primo non istituisce tout court la categoria del religioso, che presenta una strutturazione cultuale esogena, pur potendola permeare. Henri De Lubac (1979, 51), a questo proposito, nota che "senza pronunciarsi per il sì o per il no a riguardo della radice mistica di ogni religione, è possibile affermare che attualmente non ogni religione è mistica, non più di quanto ogni mistica sia religiosa"; oltre a ciò, indica il rapporto tra filosofia morale, religione e mistica.

De Lubac problematizza l'integrazione della morale nella mistica, la quale non può essere messa a fuoco da una antropologia -e si aggiunge da una pedagogiaa lente intellettualista, psicologista e moralista. Nell'ámbito della filosofia della formazione sarebbe interessante ripensare la specificazione di De Lubac per poter addentrarsi nella scissura tra ciò che è spirituale e ciò che appartiene allo psichismo, in merito alla formazione e alla trasformazione dell'uomo. Il fine sarebbe quello di non scivolare nella indiscriminazione dei due ámbiti. Alla dimensione psicologica "non è accessibile quella profondità che è la Trascendenza umana, quella zona diversamente misteriosa dove dimora lo slancio verso Dio, l'incontro con Dio" (De Lubac, 1979, 163). Non approfondendo l'indicazione specifica di Heribert Fischer

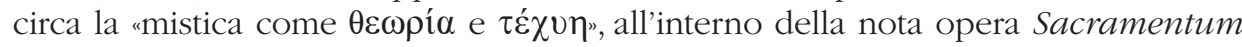
Mundi. Theologisches Lexicon für die Praxis-4 Bände e nell'ottica di una teologia della mistica, si intende precisare che la mistica dovrebbe riguardare anche l'antropologia pedagogica, poiché concerne l'uomo il quale, ricevendo la grazia divina, misticamente si forma in quel Luogo, che è Dio. Nel contesto propriamente cristiano, alla mistica speculativa tedesca - situata storicamente tra XII e XV secolo e sostanziata da un fascio di nessi anche a matrice neoplatonica e dionisiana- va associata l'intensità della mistica dell'essenza carmelitana spagnola del XVI secolo (implicitamente alimentata dalla prima) e la mistica dell'alterità soprattutto nel XIX secolo.

Al di là delle distinzioni, la mistica rimane esperienza del Mistero nel Grund dell'essenza umana e sussiste in quanto inesplicabile prossimità con il totalmente Lontano che è, nell'ottica di Margherita Porete, totalmente Vicino. In altri termini, la mistica è esperienza dello Spirito, il quale «è conoscenza, è perciò il sempre identico muoversi tra un altro sempre trascendente e un identico sempre immanente" (Vannini, 2000, 9). Delineare la mistica come esperienza del Mistero di Dio significa coglierla nel suo tropismo, in quanto radicale esperienza dell'Amore assoluto verso 
l'uomo. Allo stesso modo in cui l'amore creaturale -reso emblematico dagli amanti del Cantico veterotestamentario- è un mistero che non si conosce ma si riconosce, così ogni mistico distingue la tenebra e la luce in relazione all'Amato. Il teologo e poeta San Juan de la Cruz, formatosi all'Università di Salamanca tra il 1564 e il 1568, interpreta la medesima dialettica tra assenza e presenza di Dio in termini estremi: "Vivo sin vivir en mí, / y de tal manera espero, / que muero porque no muero".

Nondimeno nelle Opere del dottore della Chiesa, Santa Teresa de Ávila ${ }^{13}$ ritroviamo la stessa ieràtica essenzialità sanjuanista e la fruizione linguistica di una timbrica mistica quasi sovrapponibile. Per tali mistici carmelitani, riconoscere la vibrazione del divino in ogni realtà equivale alla ricerca di sé nell'Amato e dell'Amato in sé -in quanto "forte come la morte è l'amore" (Ct 8,6)-. La formazione spirituale implica sempre il riconoscimento ontologico della propria verità umana. Riconoscere il non-velato significa, da parte dell'uomo che si forma in Dio, ammettere il Mistero senza poterlo svelare ed abbandonare la volontà di rappresentarsi se non in quanto immagine creata dell'Increato.

In senso pedagogico, l'uomo spirituale viene liberato dall'unico Maestro, dalla Parola incarnata. È la Verità che rende liberi (Gv 8,31-32) di scegliere -nel bene- l'organizzazione della propria esistenza. A partire dal 1500, la dinamica del discernimento spirituale viene attivata da un complesso organico di esercizi interiori (a funzione ascetica, elettiva e unitiva) che trova la sua summa negli Ejercicios espirituales di Íñigo López de Loyola ${ }^{14}$, la cui azione pedagogica consiste nella cómpito spirituale di «togliere da sé tutti i legami disordinati e, dopo averli tolti, di cercare e trovare la volontà divina".

La formazione spirituale si specifica, dunque, nel corrispondere alla volontà amorevole di Dio -che è "forma delle forme" (Cusano, 1998, 130)-.

Emerge, allora, l'idea di forma nel senso ebraico-maimonideo di temunah. Nell'ambito neotestamentario, il Creatore è Mistero innominabile e invisibile, eppure nominato e reso visibile nel Verbo incarnato che era presso Dio e che è Dio (Gv 1,1).

L'inscrizione del mistero dell'uomo nel Mysterium Christi, in relazione all'idea di Dio come praecisio, risulta eccellente nella "matematica mistica" cusaniana del XV secolo. In seguito all'espansione dello scotismo ed evocando il Corpus Hermeticum (Ermete Trismegisto, 2005), Niccolò Cusano (1998, 99) passa "dalla sfera infinita all'esistenza attuale di Dio". L'autore della Docta ignorantia sottolinea che al "massimo assoluto" non può adattarsi nessun nome, in concordanza a quel mistero per cui "il nome di Dio è "Dio"” (ibid., 102). Anche nella tradizione ebraica, nel linguaggio originario e mistico della rivelazione divina, "prima della creazione

13. Teresa de Ávila (1954).

14. Cf. De Dalmases (1979). La traduzione italiana di tale biografia di formazione è datata 1984 e riconducibile alla casa editrice Jaka Book di Milano. 
vi erano solo Dio e il suo Nome" (Scholem, 2005, 27). Dopo la creazione rimane

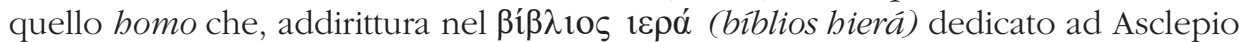
(Ermete Trismegisto, 2005, 522-523), viene definito "magnum miraculum [...] animal adorandum atque honorandum" ("grande miracolo [...] vivente degno di rispetto e di onore").

La pedagogia può fronteggiare il mysterium hominis nel Mistero di Dio. Se l'uomo è la sua formazione, se la formazione si configura come mistero e se l'essere umano si delinea in quanto mistero creato, allora la filosofia della formazione non può certo ignorare quel Mistero increato, per cui -nella cultura ebraico-cristiana"l'umanità ha indissolubilmente messo le sue radici in alto" (Cusano, 1998, 180).

Il Misterium Dei si celebra nel mistero dell'uomo. Infatti, proprio dalle profondità del più oscuro e fitto Mistero, uno dei più grandi mistici, lo spagnolo Juan de la Cruz $^{15}$ : canta il desiderio di Dio nell'ultima esperanza dello slancio umano: "Cuanto más alto subía / deslumbróseme la vista, / y la más fuerte conquista / en oscuro se hacía; / mas, por ser de amor el lance, / di un ciego y oscuro salto, / y fui tan alto, tan alto, / que le di a la caza alcance".

\section{Bibliografia}

Agathias, L. (1977) La mistica greca, en Davy, M. M. (ed.). Encyclopédie des mystiques: Chamanisme, grecs, gnose, christianisme primitif. Paris, Éd. Robert Laffont et Éd. Jupiter.

Aletti, J. N. (2005) Mistero. A. Teologia biblica, in Lacoste, J. Y. (ed.). Dizionario critico di teologia. Roma, Borla-Città Nuova, 856-857.

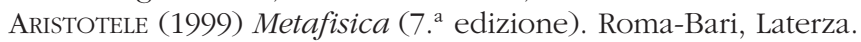

Benjamin, W. (1972-1989) Gesammelte Schriften. Frankfurt a. M., Suhrkamp.

Bologna, C. (1992) Flatus vocis. Metafisica e antropologia della voce. Bologna, il Mulino.

BOuYer, L. (1986) Mysterion. Du mistère à la mystique. O.E.I.L.

Catullo, G. V. (1999) Le poesie. Carmina (2. ${ }^{\text {a }}$ edizione). Roma, Newton Compton.

Colli, G. (1975) La nascita della filosofia. Milano, Adelphi.

Cusano, N. (1998) La dotta ignoranza. Roma, Città Nuova.

Davy, M. M. (ed.) (2000) Esperienze mistiche in Oriente e in Occidente. Dottrine e profili. Città del Vaticano, Libreria Editrice Vaticana.

De Dalmases, C. (1979) El padre maestro Ignacio. Madrid, Biblioteca de Autores Cristianos.

De Lubac, H. (1979) Mistica e mistero cristiano. La fede cristiana. Milano, Jaka Book.

15. Solo a titolo indicativo, tra le molte, si ricordano solo alcune opere classiche del Santo cf. Obras de San Juan de la Cruz doctor de la Iglesia (4. ${ }^{\mathrm{a}}$ ed.). Madrid, Apostolato de la Prensa, 1943. In particolare, nel 1955, Cántico espiritual. Introducción de Fortunato de Jesús Sacramentado,

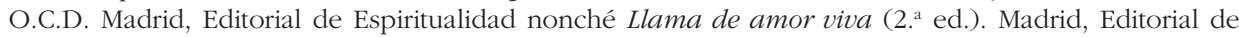
Espiritualidad; a cui si aggiunge San Juan de la Cruz - Teresa - S. Teresita. Lira mistica. Madrid, Editorial de Espiritualidad. Per le questioni di pedagogia, oltre Jiménez Duque che ha studiato La pedagogía de San Juan de la Cruz, anche SAAVEDRA (1943). 
LA QUESTIONE DEL MISTERO UMANO NELLA FILOSOFIA DELLA FORMAZIONE

Derrey, N. (2005) Mistero. B. Teologia liturgica e sacramentarla, in Lacoste, J. Y. (ed.). Dizionario critico di teologia. Roma, Borla-Città Nuova, 857-859.

Dostoevskij, F. M. (1964) I fratelli Karamàzov. Torino-Milano, Einaudi-Mondadori.

- (1995) Il sogno di un uomo ridicolo e La mite. Due racconti fantastici. Roma, Newton Compton.

ECKHART, Meister (1999) Il nulla divino. Milano, Mondadori.

Trismegisto, Hermes (2005) Corpus Hermeticum (testo greco, latino e copto). Milano, Bompiani.

Evdokìmov, P. N. (1972) L'art de l'icône. Théologie de la beauté. Paris, Desclée de Brouwer.

Gennari, M. (1995a) Modernità e mistero. Teologia, pneumatologia e filosofia dell'educazione, Studi Europei, III, 203-251.

- (1995b) Storia della Bildung. Formazione dell'uomo e storia della cultura in Germania e nella Mitteleuropea. Brescia, La Scuola.

- (2001) Filosofia della formazione dell'uomo. Milano, Bompiani.

- (2007) Filosofia del pensiero. Genova, Il melangolo.

Gennari, M. y KaIser, A. (2000) Prolegomeni alla Pedagogia Generale. Milano, Bompiani.

Heidegger, M. (1956) Was ist das-die philosophie? Günther Neske, Pfullingen.

- (1959) Gelassenheit. Günther Neske, Pfullingen.

- (1983) Die Grundbegriffe der Metaphisik. Welt-Endlichkeit-Einsamkeit. Frankfurt a. M., Klostermann.

- (1999) Sull'essenza della verità. Roma, Armando, 81.

Hölderlin, F. (1996) Scritti di estetica. Milano, Mondadori.

Huot de Longchamp, M. (2005) Mistica, in Lacoste, J. Y. (ed.). Dizionario critico di teologia. Roma, Borla-Città Nuova, 859-865.

KAISER, A. (1998) Gnoseologia dell'educazione. 1. Lo statuto antropologico dell'essere spirituale. Brescia, La Scuola.

KierKegAaRd, S. (1991) La malattia mortale. Milano, Mondadori.

Montinari, G. (1998) L'agnello e la scure. Viaggio Psicoantropologico alle radici. Milano, FrancoAngeli, 116-117.

Lacoste, J. Y. (ed.) (1998) Dictionnaire critique de théologie. Paris, Presses Universitaires de France.

Ottonello, P. P. (1967) Bibliografia di S. Juan de la Cruz. Roma, Teresianum.

Pessoa, F. (2002) Poesie esoteriche. Milano, TEA.

Platone (1995) Eutifrone (9. ${ }^{\text {a }}$ edizione). Brescia, La Scuola.

PluTARCO (2006) L'arte di saper ascoltare. Roma, Newton Compton.

Price, S. (1999) Religions of the Ancient Greeks. Cambridge, Cambridge University.

- (2002) Le religioni dei greci. Bologna, il Mulino, 117-136.

RAHNER, K. (1967-1969) Sacramentum mundi. Theologisches Lexikon für die Praxis-4 Bände. Freiburg im Breisgau, Herder KG.

RANK, O. (1914) Der Doppelgänger. Wien-Leipzig.

Rimbaud, A. (1975) Poesie e Illuminazioni. Milano, Mondadori, 29.

Rosenzweig, F. (2000) Ebraismo, Bildung e filosofia della vita. Firenze, La Giuntina.

SAavedra, M. (1943) Las maneras pedagógicas de San Juan de la Cruz, Revista de Espiritualidad, Madrid, II, n. ${ }^{\circ}$ 7, 91-101.

Scholem, G. (1970) Der Name Gottes und die Sprachtheorie der Kabbala. Frankfurt a. M., Suhrkamp. 
SiLEsIus, A. (1675) Cherubinischer Wandersmann, oder Geist-Reiche Sinn-und SchlußReime zur Göttlichen beschauligkeit anleitende. Von dem Urheber aufs neue übersehn, und mit dem Sechsten Buche vermehrt, den Liebhabern der geheimen Theologie und beschaulichen Lebens zur Geistlichen Ergötzligkeit zum andernmahl herauß gegeben. Glatz.

SolA, G. (2003) Umbildung. La "trasformazione" nella formazione dell'uomo. Milano, Bompiani.

Teresa de Ávila (1954) Obras Completas. Madrid, Biblioteca de Autores Cristianos.

VAléry, P. (1942) Mauvaises Pensées et autres. Paris, Gallimard.

VANNInI, M. (1999) Il volto del Dio nascosto. Milano, Mondadori.

- (2000) Introduzione alla mistica. Morcelliana, Brescia. 